\title{
A Simple Gas-Kinetic Model for Dilute and Weakly Charged Plasma Micro-Jet Flows
}

\author{
Shiying Cai and Chunpei Cai *
}

check for

updates

Citation: Cai, S.; Cai, C. A Simple Gas-Kinetic Model for Dilute and Weakly Charged Plasma Micro-Jet Flows. Fluids 2021, 6, 250. https:// doi.org/10.3390/fluids6070250

Academic Editor: Rene Van Hout

Received: 30 May 2021

Accepted: 29 June 2021

Published: 7 July 2021

Publisher's Note: MDPI stays neutral with regard to jurisdictional claims in published maps and institutional affiliations.

Copyright: (c) 2021 by the authors. Licensee MDPI, Basel, Switzerland. This article is an open access article distributed under the terms and conditions of the Creative Commons Attribution (CC BY) license (https:/ / creativecommons.org/licenses/by/ $4.0 /)$.
Department of Mechanical Engineering-Engineering Mechanics, Michigan Technological University, Houghton, MI 49931, USA; shiyingc@mtu.edu

* Correspondence: ccai@mtu.edu; Tel.: +1-575-915-5687

\begin{abstract}
This paper presents a simple model for slightly charged gas expanding into a vacuum from a planar exit. The number density, bulk velocity, temperature, and potential at the exit are given. The electric field force is assumed weaker than the convection term and is neglected in the analysis. As such, the quasi-neutral condition is naturally adopted and the potential field is computed with the Boltzmann relation. At far field, the exit degenerates as a point source, and simplified analytical formulas for flow and electric fields are obtained. The results are generic and offer insights on many existing models in the literature. They can be used to quickly approximate the flowfield and potential distributions without numerical simulations. They can also be used to initialize a simulation. Based on these results, more advanced models may be further developed.
\end{abstract}

Keywords: gaskinetic theory; free molecular flow; jet; plasma; electrostatics

\section{Introduction}

Highly dilute neutral or slightly charged gaseous jet is a fundamental problem with many applications. In the literature, many comprehensive investigations are reported on neutral jet flows. However, due to the complex physics, there are less work reports on slightly charged gaseous jets. Most work relies on numerical simulations, and theoretical models are rare. This paper aims to provide such a theoretical model with numerical validations.

The paper continues as follows. Section 2 reviews related past work, Section 3 presents recent modeling work on potentials and farfield properties, Section 4 provides validations and discussions, and Section 5 summarizes the work with a few conclusions.

\section{Past Work on Dilute Jet Flows}

For a dilute jet expanding into a vacuum, the past work concentrates on two scenarios: with or without charges. The scenario without charges is more fundamental. Both flows are widely observed, e.g., plume impingement, in the astrophysical context, negatively charged dust particles in cometary tails expanding into the interplanetary space [1,2], or the expansion of the solar wind plasma into the wake region of inert objects, such as asteroids or the moon [3]. Woronowicz [4] described validation effort to assess the viability of applying a set of analytic point source transient free molecule equations to model behavior ranger from molecular effusion to rocket plumes. For numerical simulations, particle methods are widely used to simulate the second problem due to the complex physics. For example, the direct simulation Monte Carlo (DSMC) method [5] is commonly adopted to simulate neutral gas flows, and the Particle-In-Cell (PIC) method [6] for charged gas flows. For example, Roy, Hastings and Gatsonis [7] proposed an asymmetric model of the plume and back-flow contamination from an ion-thruster plume, and performed hybrid PIC simulations to simulate charge-exchange efflux ions. We can only name a few.

Many past theoretical studies or modeling work focused on one-dimensional, unsteady flows, most of them adopted hydrodynamics approaches, and most of them pro- 
vided flow and electric field results. Due to the complexities, it is challenging to obtain complete solutions for two-dimensional and three-dimensional flows and the results are scarce. In the past decade, thorough investigations (e.g., [8,9]) on the first problem with a gaskinetic method were performed. The planar scenario is illustrated in Figure 1. A collisionless and neutral gas fires from a planar exit with a semi-height $H$. At the exit, the gas has a number density $n_{0}$, a bulk velocity $U_{0}$, and a temperature $T_{0}$. The flow field can be represented either with a Cartesian coordinate system $(X, Y)$, or equivalently a polar coordinate system $\left(r, \alpha_{0}\right)$. Several key angles are defined in Figure 1: $\tan \theta_{1}=(Y-H) / X, \tan \theta_{2}=(Y+H) / X$, $\tan \theta_{3}=H / X$ and $\tan \alpha_{0}=Y / X$. A gaskinetic method was adopted, and the theoretical flowfield formulas include density $n(X, Y)$, velocity components $U(X, Y)$ and $V(X, Y)$, and temperature $T(X, Y)$. The flowfield properties at a specific point $P(X, Y)$ are:

$$
\begin{gathered}
\frac{n(X, Y)}{n_{0}}=\frac{e^{-S_{0}^{2}}}{2 \pi} \int_{\theta_{1}}^{\theta_{2}} A\left(S_{0} \cos \theta\right) d \theta, \\
\frac{U(X, Y)}{\sqrt{2 R T_{0}}}=\frac{e^{-S_{0}^{2}}}{2 \pi} \frac{n_{0}}{n(X, Y)} \int_{\theta_{1}}^{\theta_{2}} B\left(S_{0} \cos \theta\right) \cos \theta d \theta, \\
\frac{V(X, Y)}{\sqrt{2 R T_{0}}}=\frac{e^{-S_{0}^{2}}}{4 S_{0} \sqrt{\pi}} \frac{n_{0}}{n}\left(t_{1} e^{t_{1}^{2}}\left[1+\operatorname{erf}\left(t_{1}\right)\right]-t_{2} e^{t_{2}^{2}}\left[1+\operatorname{erf}\left(t_{2}\right)\right]\right)=\frac{e^{-S_{0}^{2}}}{2 \pi} \frac{n_{0}}{n} \int_{\theta_{1}}^{\theta_{2}} B(t) \sin \theta d \theta, \\
\frac{T(X, Y)}{T_{0}}=\frac{2}{3} \frac{e^{-S_{0}^{2}}}{\pi} \frac{n_{0}}{n(X, Y)} \int_{\theta_{1}}^{\theta_{2}} C\left(S_{0} \cos \theta\right) d \theta-\frac{U^{2}(X, Y)+V^{2}(X, Y)}{3 R T_{0}},
\end{gathered}
$$

where $S_{0}=U_{0} / \sqrt{2 R T_{0}}, t=S_{0} \cos \theta$,

$$
\begin{aligned}
& A(t)=1+\sqrt{\pi} t e^{t^{2}}[1+\operatorname{erf}(t)], \quad B(t)=t+\sqrt{\pi}\left(\frac{1}{2}+t^{2}\right) e^{t^{2}}[1+\operatorname{erf}(t)], \\
& C(t)=\frac{3}{4}+\frac{t^{2}}{2}+\sqrt{\pi}\left(t+\frac{t^{3}}{2}\right) e^{t^{2}}[1+\operatorname{erf}(t)] \text {. }
\end{aligned}
$$

There are two relations among them, $d A(t) / d t=2 B(t)$ and $d B(t) / d t=4 C(t)-A(t)$. The above results can be also written in a polar coordinate system $(r, \alpha)$, which is more convenient for farfield flow solutions.

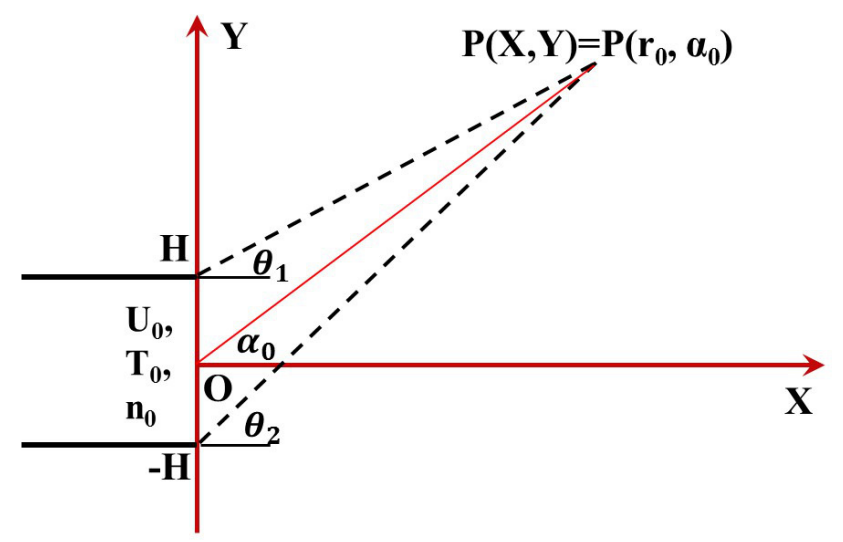

Figure 1. Illustrations for the problem of a neutral and collision-less jet expanding into a vacuum.

\section{Potential and Farfield Approximations}

One example for the second problem is electro-sprays in a vacuum condition, e.g., for space propulsion. The related flow stages for an electro-spray flow with ions and charged droplets $[10,11]$ include a liquid meniscus, a Taylor cone, a cone-jet connection, a jet, a jet end (with a diameter of micro-meters) where ions evaporate into a vacuum, and form a final spray with a size of centimeters or beyond. The jet end is critical but it is rather small to be recognized in computational fluid simulations where usually the mesh size can not 
be micro-meters. As such, sub-grid modeling work on the jet end and plume flows must be done to resolve this issue. A recent paper [12] has reported the development of such an axisymmetric model, and the purpose of this paper is to complete the counterpart model for a two-dimensional flow scenario. The fundamental assumptions are still the exit speeds are very high, and the local potential changes are very mild.

Translational temperatures. For high speed gaseous neutral plume flows, the local translational temperatures $T_{x}(X, Y)$ and $T_{y}(X, Y)$ along the stream and transverse directions can be quite different and their effects on charges can be quite different; hence, the analytical expressions for $T_{x}$ and $T_{y}$ are studied first. Following the same vein to obtain Equations (1)-(4), they are obtained and presented as follows:

$$
\begin{aligned}
& \frac{T_{x}(X, Y)}{T_{0}}=\frac{e^{-S_{0}^{2}}}{\pi} \frac{n_{0}}{n(X, Y)} \int_{\theta_{1}}^{\theta_{2}} D\left(S_{0} \cos \theta\right) \cos ^{2} \theta d \theta-\frac{U^{2}(X, Y)}{R T_{0}}, \\
& \frac{T_{y}(X, Y)}{T_{0}}=\frac{e^{-S_{0}^{2}}}{\pi} \frac{n_{0}}{n(X, Y)} \int_{\theta_{1}}^{\theta_{2}} D\left(S_{0} \cos \theta\right) \sin ^{2} \theta d \theta-\frac{V^{2}(X, Y)}{R T_{0}}, \\
& D(t)=1+t^{2}+\sqrt{\pi}\left(\frac{3}{2} t+t^{3}\right) e^{t^{2}}[1+\operatorname{erf}(t)]=2 C(t)-\frac{1}{2} A(t) .
\end{aligned}
$$

As shown, there are " $\cos ^{2} \theta$ " and " $\sin ^{2} \theta$ " in the integrands, and the corresponding velocity components are different as well.

Potential and electric fields. It is well known that, plasma flows are described by the kinetic equation for the velocity distribution function $f$ for ions:

$$
\frac{\partial f}{\partial t}+\nabla_{x} f \cdot \vec{v}-\left(\nabla_{v} f \cdot \nabla_{x} \phi\right) \frac{e}{M}=0,
$$

where $e$ is the unit charge, and $M$ is the ion mass. By comparing the 2nd and the 3rd terms in the above equation, under certain conditions, such as the velocity magnitude $|v|$ is very large or the bulk velocity $U_{0}$ is very large, and the change in local potential $\phi$ is very mild, then the 3rd term is small and can be neglected. As such, the plasma flows can be treated as quasi-neutral gas flows, and it may be feasible to use the above gaskinetic approach to study the plasma plume flows.

In this work, we introduce the widely used Boltzmann relations for electrons in a quasi-neutral plasma flows:

$$
\phi(X, Y)=\phi_{r e f}+\frac{k T_{r e f}}{e} \ln \frac{n(X, Y)}{n_{r e f}}
$$

where subscript ${ }_{r e f}$ represents related reference values, $k$ is the Boltzmann constant, and $e$ is the standard electron charge. With almost the same amount of electron and ion number densities, $n_{e} \approx n_{i}$, and an ion and an electron have the same amount of charge and the same electric forces, a crude approximate condition $T_{e} \approx T_{i}$ is obtained.

With the Boltzmann relation, the potential and temperature at the exit, $\phi_{0}$ and $T_{0}$, are chosen as the reference values in Equation (10), and the local density formula, Equation (1) for $n(X, Y)$, is used to compute the local potential $\phi(X, Y)$. This is a direct and probably the simplest method, and the expression is:

$$
\frac{\phi(X, Y)}{\phi_{r e f}}=1+\frac{k T_{r e f}}{e \phi_{r e f}}\left(\ln \int_{\theta_{1}}^{\theta_{2}} A\left(S_{0} \cos \theta\right) d \theta-\ln (2 \pi)-S_{0}^{2}\right) .
$$

From Equation (11), with the aid of the Leibniz integral rule [13], and chain rule for derivations, the corresponding electric field components at point $P(X, Y)$ can be derived as:

$$
E_{x}=-\frac{\partial \phi}{\partial X}=\frac{k T_{r e f}}{e X \int_{\theta_{1}}^{\theta_{2}} A(t) d \theta}\left(\frac{A\left(t_{2}\right)}{2} \sin 2 \theta_{2}-\frac{A\left(t_{1}\right)}{2} \sin 2 \theta_{1}-2 S_{0} \int_{\theta_{1}}^{\theta_{2}} B(t) \sin ^{2} \theta \cos \theta d \theta\right) \text {, }
$$




$$
E_{y}=-\frac{\partial \phi}{\partial Y}=\frac{k T_{r e f}}{e X \int_{\theta_{1}}^{\theta_{2}} A(t) d \theta}\left(A\left(t_{1}\right) \cos ^{2} \theta_{1}-A\left(t_{2}\right) \cos ^{2} \theta_{2}+2 S_{0} \int_{\theta_{1}}^{\theta_{2}} B(t) \sin \theta \cos ^{2} \theta d \theta\right) .
$$

Correspondingly, $E_{r}\left(r, \alpha_{0}\right)$ and $E_{\alpha_{0}}\left(r, \alpha_{0}\right)$ can be derived as:

$$
\begin{aligned}
E_{r}\left(r, \alpha_{0}\right) & =E_{x}(X, Y) \cos \alpha_{0}+E_{y}(X, Y) \sin \alpha_{0} \\
E_{\alpha_{0}}\left(r, \alpha_{0}\right) & =E_{y}(X, Y) \cos \alpha_{0}-E_{x}(X, Y) \sin \alpha_{0} .
\end{aligned}
$$

Far Field Properties. At a farfield point $P(X, Y)$ in a Cartesian coordinate system, or $P\left(r_{0}, \alpha_{0}\right)$ in a polar coordinate system, the distance to the coordinate origin, $r_{0}=\sqrt{X^{2}+Y^{2}}$ is assumed to be much larger than the exit width, $2 H$, the angle is $\alpha_{0}=\arctan (Y / X)$, and the exit degenerates as one source point. The solid angle subtended by the point and the two exit lips, $\Delta \theta=\theta_{2}-\theta_{1}$, is quite small, and there are two following relations:

$$
\Delta \theta=\operatorname{atan} \frac{(2 H) X}{X^{2}+Y^{2}-H^{2}} \approx \frac{2 H \cos \alpha_{0}}{r_{0}}, \operatorname{erf}\left(t_{2}\right) \approx \operatorname{erf}\left(t_{1}\right)-\frac{2 S_{0} \sin \theta_{1}}{\sqrt{\pi}} \Delta \theta e^{-t_{1}^{2}} .
$$

With these relations, the simplified number density, velocity components, temperature components, potentials, the electric field components, are derived from Equations (1)-(7). The results are listed as follows, with $0 \leq \alpha_{0}<\pi / 2$ :

$$
\begin{gathered}
\frac{n_{1}(X, Y)}{n_{0}} \approx \frac{e^{-S_{0}^{2}}}{2 \pi} \frac{(2 H) \cos \alpha_{0}}{r_{0}} A\left(t_{0}\right), \\
\frac{U_{1}(X, Y)}{\sqrt{2 R T_{0}}} \approx \frac{B\left(t_{0}\right)}{A\left(t_{0}\right)} \cos \alpha_{0}, \frac{V_{1}(X, Y)}{\sqrt{2 R T_{0}}} \approx \frac{B\left(t_{0}\right)}{A\left(t_{0}\right)} \sin \alpha_{0}, \\
\frac{T_{1}(X, Y)}{T_{0}} \approx \frac{4}{3} \frac{C\left(t_{0}\right)}{A\left(t_{0}\right)}-\frac{2}{3} \frac{B^{2}\left(t_{0}\right)}{A^{2}\left(t_{0}\right)}, \\
\frac{T_{1 x}}{T_{0}} \approx 2 \frac{A\left(t_{0}\right) D\left(t_{0}\right)-B^{2}\left(t_{0}\right)}{A^{2}\left(t_{0}\right)} \cos ^{2} \alpha_{0}, \frac{T_{1 y}}{T_{0}} \approx 2 \frac{A\left(t_{0}\right) D\left(t_{0}\right)-B^{2}\left(t_{0}\right)}{A^{2}\left(t_{0}\right)} \sin ^{2} \alpha_{0},
\end{gathered}
$$

where $A, B, C$ and $D$ are defined by Equations (5) and (8). They are not related with $r_{0}$, but they are functions of $t_{0}=S_{0} \cos \alpha_{0}$.

The total current within the angle smaller than $\alpha_{0}$ is computed by Equations (16) and (17):

$$
\frac{J\left(r_{0}, S_{0}, \alpha_{0}\right)}{e n_{0} r_{0} \sqrt{2 R T_{0}}} \approx \frac{e^{-S_{0}^{2}}}{\pi} \frac{H}{r_{0}} \int_{0}^{\alpha_{0}} B\left(S_{0} \cos \theta\right) \cos \theta d \theta .
$$

This formula indicates at farfield, the current within a certain angle $\alpha_{0}$ is constant. The potential field is derived from Equation (16) and the Boltzmann relation:

$$
\frac{\phi_{1}(X, Y)}{\phi_{r e f}} \approx 1+\frac{k T_{r e f}}{e \phi_{r e f}}\left(\ln \left(A\left(S_{0} \cos \alpha_{0}\right)\right)-\ln (2 \pi)-S_{0}^{2}+\ln \frac{2 H \cos \alpha_{0}}{r_{0}}\right) .
$$

The electric field components in a polar or a Cartesian coordinate are:

$$
\begin{array}{rll}
E_{r_{0}, 1} & \approx \frac{k T_{r e f}}{e r_{0}}, & E_{\alpha_{0}, 1} \approx \frac{k T_{r e f}}{e r_{0}}\left(1+2 t_{0} \frac{B\left(t_{0}\right)}{A\left(t_{0}\right)}\right) \tan \alpha_{0} \\
E_{x} \approx \frac{k T_{r e f}}{e r_{0}}\left(1-\tan ^{2} \alpha_{0}-2 t_{0} \tan ^{2} \alpha_{0} \frac{B\left(t_{0}\right)}{A\left(t_{0}\right)}\right) \cos \alpha_{0}, & E_{y} \approx \frac{k T_{r e f}}{e r_{0}}\left(2+2 t_{0} \frac{B\left(t_{0}\right)}{A\left(t_{0}\right)}\right) \sin \alpha_{0} .
\end{array}
$$

$E_{\alpha}$ and $E_{x}$ have singular values at $\alpha_{0}=\pi / 2$, because in this model, no particle can move upwards along that line. Hence, the density along $\alpha_{0}=\pi / 2$ is zero, resulting in a singularity for the potential due to the log function. This gaskinetic model shall be applied within a smaller angle range, for example, $\alpha_{0} \leq 80^{\circ}$. 
Centerline Properties. With the relations, that $Y=0, \theta_{2}=-\theta_{1}=\theta_{3}$, the corresponding results are:

$$
\begin{gathered}
\frac{n_{2}(X, 0)}{n_{0}}=\frac{e^{-S_{0}^{2}}}{\pi G\left(\theta_{3}\right)}, \\
\frac{U_{2}(X, 0)}{\sqrt{2 R T_{0}}}=\frac{1}{G\left(\theta_{3}\right)} \int_{0}^{\theta_{3}} B(t) \cos \theta d \theta, \\
\frac{T_{2}(X, 0)}{T_{0}}=\frac{4}{3 G\left(\theta_{3}\right)} \int_{0}^{\theta_{3}} C(t) d \theta-\frac{U^{2}(X, 0)}{3 R T_{0}}, \\
\frac{T_{x, 2}(X, 0)}{T_{0}}=\frac{2}{G\left(\theta_{3}\right)} \int_{0}^{\theta_{3}} D(t) \cos ^{2} \theta d \theta-U^{2}(X, 0) /\left(R T_{0}\right), \\
\frac{T_{y, 2}(X, 0)}{T_{0}}=\frac{2}{G\left(\theta_{3}\right)} \int_{0}^{\theta_{3}} D(t) \sin ^{2} \theta d \theta, \\
\phi_{2,2}(X, 0)=\phi_{r e f}(X, 0)+\frac{k T_{r e f}}{e}\left(\ln G\left(\theta_{3}\right)-S_{0}^{2}-\ln \pi\right), \\
\frac{k T_{r e f}}{e X G\left(\theta_{3}\right)}\left(\sin \theta_{3} \cos \theta_{3} A\left(S_{0} \cos \theta_{3}\right)-S_{0} \int_{0}^{\theta_{3}} B(t) \sin ^{2} \theta \cos \theta d \theta\right),
\end{gathered}
$$

where $G\left(\theta_{3}\right)=\int_{0}^{\theta_{3}} A\left(S_{0} \cos \theta\right) d \theta$. Different from most past work by other investigators, the above results are relatively complex, but they are complete and compact. Crucial parameters, $S_{0}$ and angles, are clearly included. They are obtained directly from the generalized accurate solutions.

\section{Discussions and Farfield Property Validations}

Figure 2a compares the density expressions, Equations (1) and (16), within an angle range $0 \leq \alpha_{0} \leq 90^{\circ}$. Because Equation (16) includes a factor of $(2 H) / r_{0}$, it is more convenient to plot and compare $\frac{n_{1}}{n_{0}} \frac{r_{0}}{2 H}$. The exit speed ratios are set as $S_{0}=0.5$ and $S_{0}=2.0$, the distance ratios are chosen as $r_{0} /(2 \mathrm{H})=10$ and 100. The two curves in this figure are farfield approximations, described by Equation (16), and the symbols represent the exact solutions, described by Equation (1). As shown, results from the exact and simplified far field approximations are identical. With a higher exit speed ratio, $S_{0}=2.0$, the jet core further extends along the stream direction.

Figures $2 \mathrm{~b}$ and $3 \mathrm{a}$ present normalized $\mathrm{U}$ - and V-velocity components, with the analytical expressions (Equations (2) and (3)) and farfield simplifications (Equation (17)). It is also evident that at large angles, the exact and farfield approximations have larger discrepancies. The real situation is not so severe because the density at a large $\alpha_{0}$ is small, and the related V- momentum flux at a large $\alpha_{0}$ is quite small. Equation (17) indicates the velocity components are closely related, and there is a relation, $U(X, Y) / V(X, Y)=\tan \alpha_{0}$. The exit is a point source, and particles from the exit can be considered shooting outwards without deflections. 


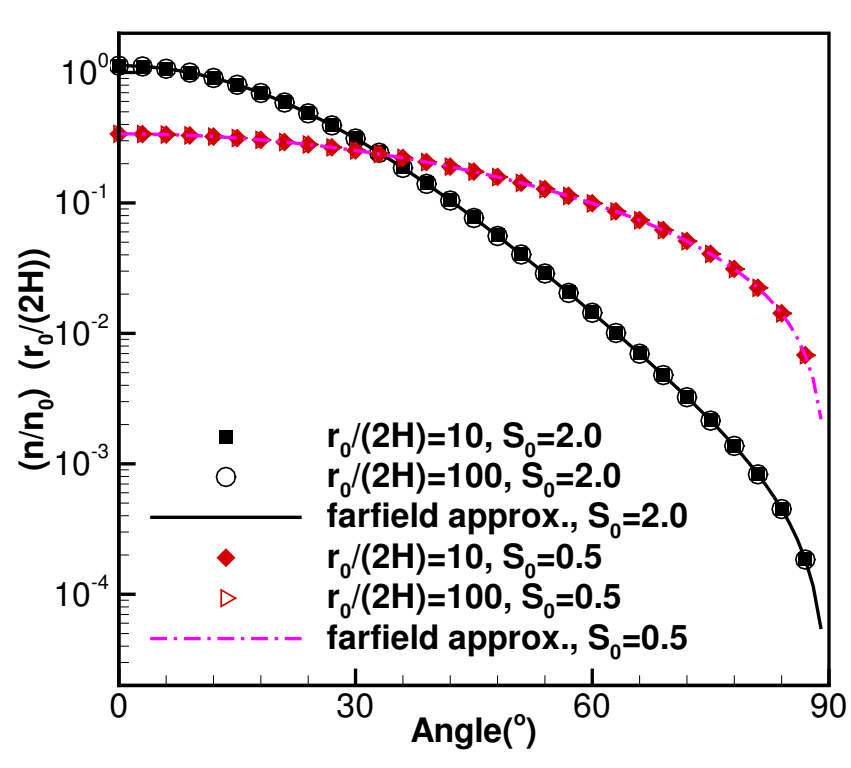

(a)

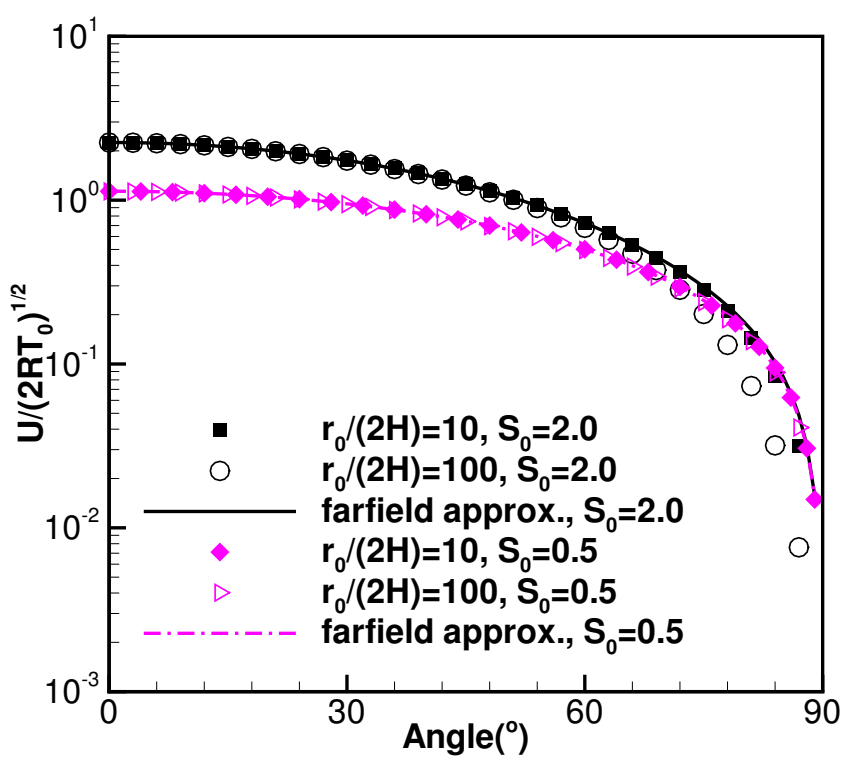

(b)

Figure 2. (a) Normalized farfield density profiles, $\frac{n_{1}(X, Y)}{n_{0}}\left(\frac{r_{0}}{2 H}\right)$; (b) Normalized farfield U-velocity component profiles, $\frac{U_{1}(X, Y)}{\sqrt{2 R T_{0}}}$.

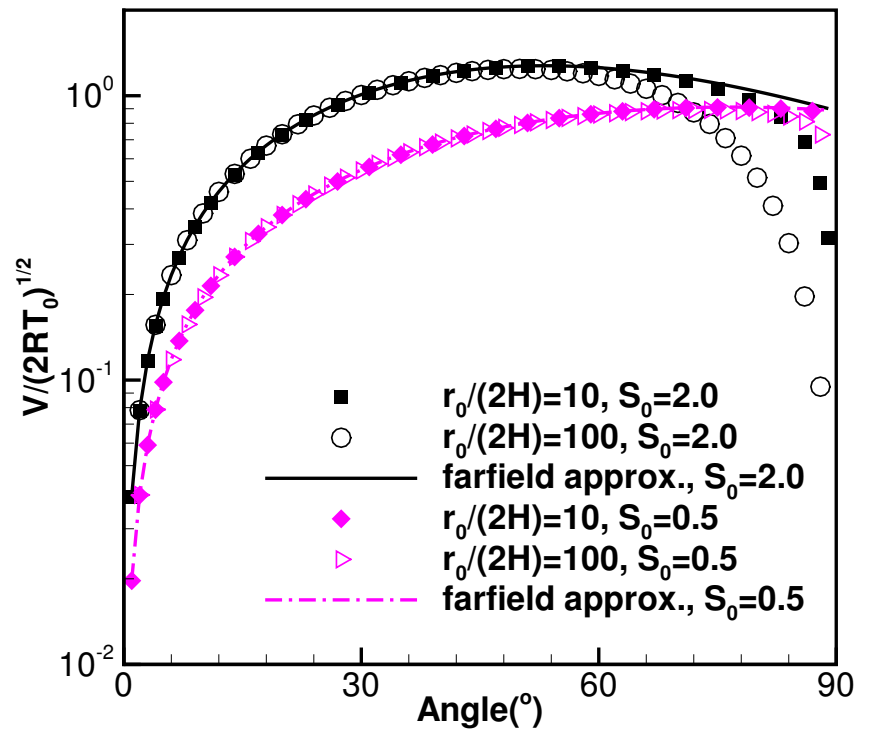

(a)

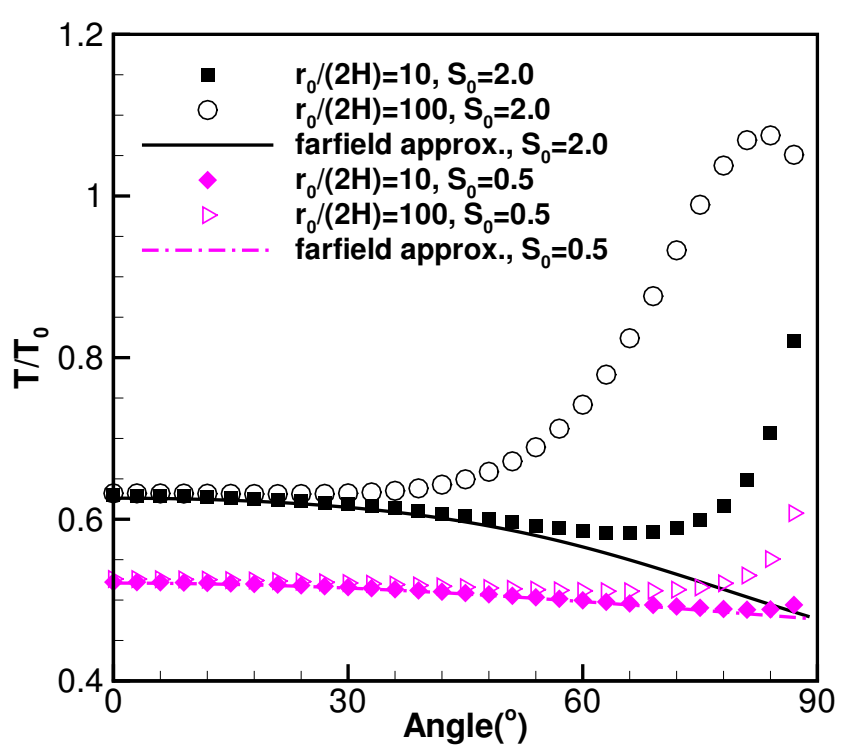

(b)

Figure 3. (a) Normalized farfield V-velocity component profiles, $\frac{V_{1}(X, Y)}{\sqrt{2 R T_{0}}} ;(\mathbf{b})$ Normalized farfield temperature profiles, $\frac{T_{1}(X, Y)}{T_{0}}$.

Figures $3 b$ and $4 a, b$ compare $T(X, Y), T_{x}(X, Y)$ and $T_{y}(X, Y)$, predicted by Equations (4), (6), (7), (18) and (19). Within the jet core, the agreements among the analytical and the farfield approximations are satisfying, and with a larger angle $\alpha_{0}$, the discrepancy increases. The largest discrepancy in $T$ is due to the related $T_{y}$ which further traces back to the $\mathrm{V}$-velocity component. However, for high speed plumes, for many situations, the plume core can be defined as a small region [14] within $\alpha_{0} \leq 30^{\circ}$. As such, it is proper to utilize these temperature expressions to evaluate local temperature fields. 


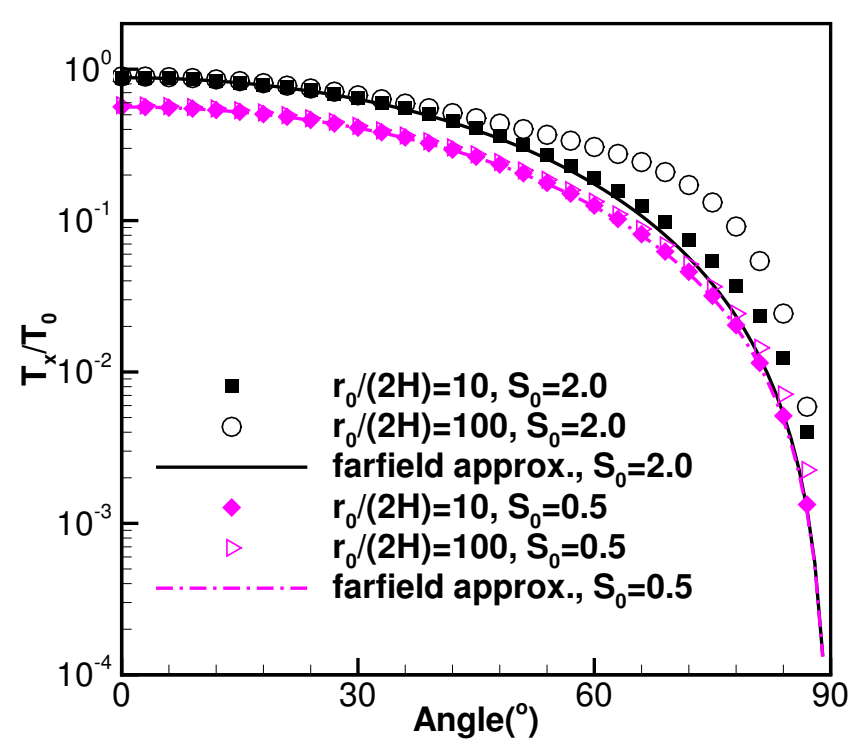

(a)

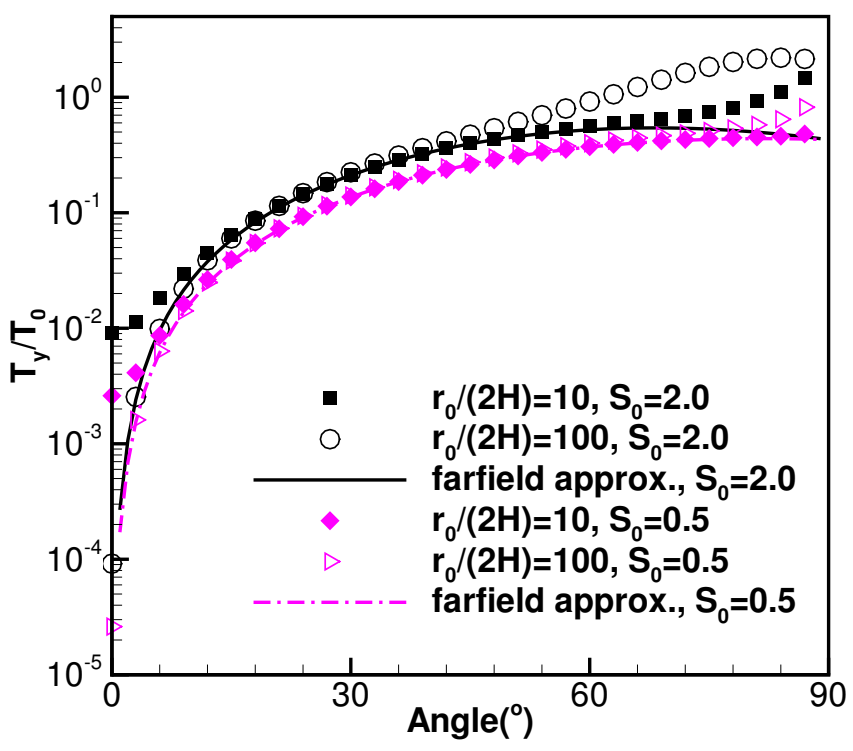

(b)

Figure 4. (a) Normalized farfield temperature profiles, $\frac{T_{x}(X, Y)}{T_{0}} ;(\mathbf{b})$ Normalized farfield temperature profiles, $\frac{T_{y}(X, Y)}{T_{0}}$.

Figure 5a shows normalized farfield number density flux $n V_{r}$ at a constant radius $r_{0} /(2 H)=10$. The profiles are similar to those for density and but this property is more helpful. In general, the profiles are steeper at the centerline because the centerline velocity is much larger.

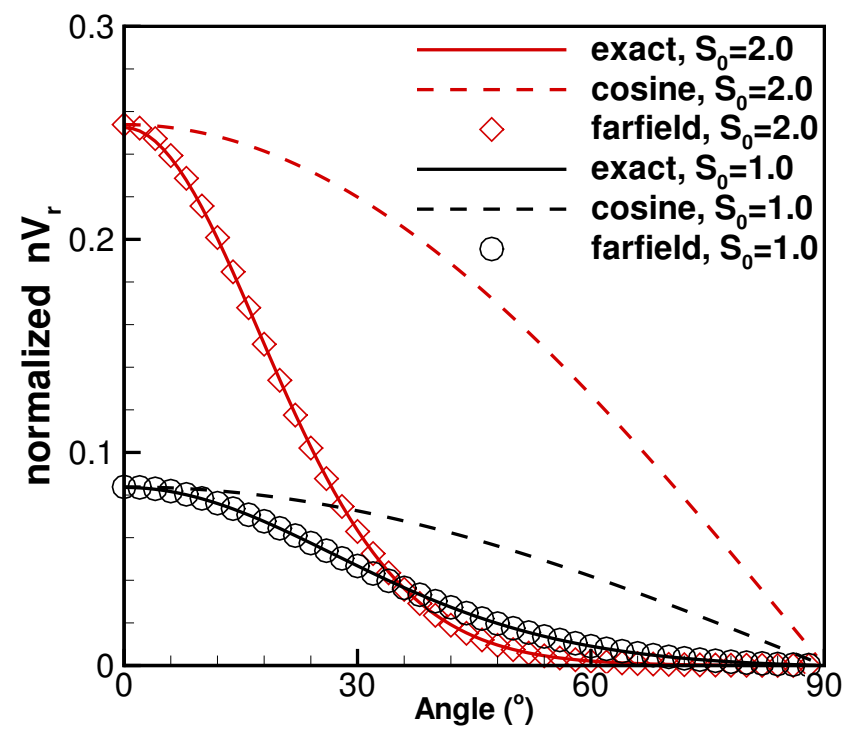

(a)

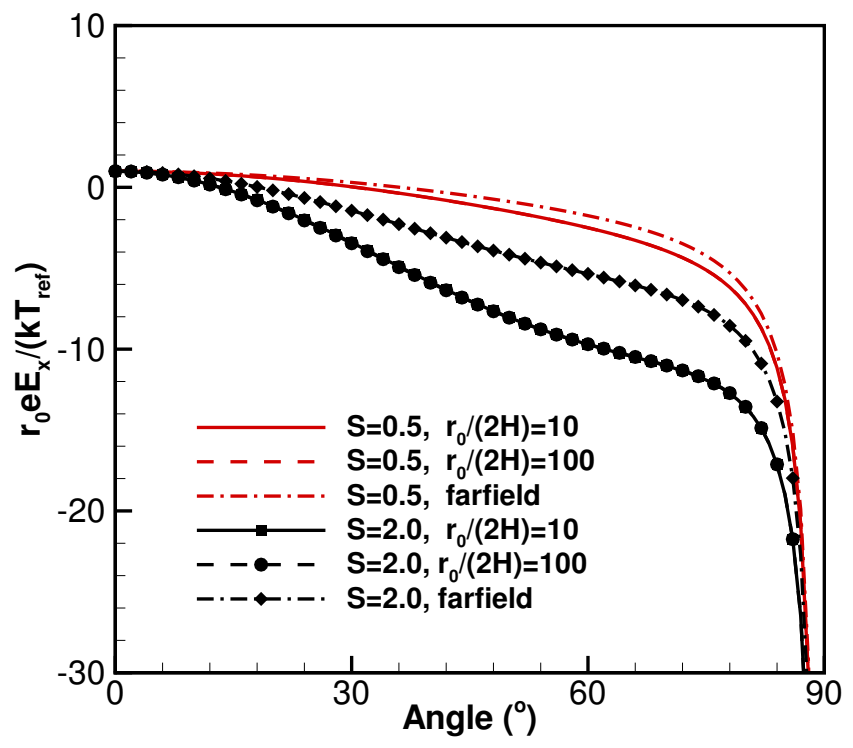

(b)

Figure 5. (a) Density flux profiles, $r_{0} /(2 H)=10 ;(\mathbf{b})$ Normalized electric field component, $e r_{0} E_{x} /\left(k T_{0}\right)$, at different angles.

Figure $5 \mathrm{~b}$ shows the normalized exact and farfield electric field component along the X-direction, at locations $r_{0} /(2 H)=10$ and 100, and $S_{0}=0.5$ and 2.0, correspondingly. The curves are computed with Equations (12) and (22). For the exact solutions and specific $S_{0}$ value, the solutions for $r_{0} /(2 H)=10$ and 100 coincident. There are appreciable difference between the exact and farfield approximations, and the deviations depend on the $S_{0}$ values. However, there is no doubt that they share the same trend. 
Figure 6a shows farfield electric field component along the Y-direction, at locations $r_{0} /(2 H)=10$ and 100, and $S_{0}=0.5$ and 2.0, correspondingly. The curves are computed with Equations (13) and (22). Similar patterns to the $E_{x}$ direction results, about the deviations and the same trends are evident.

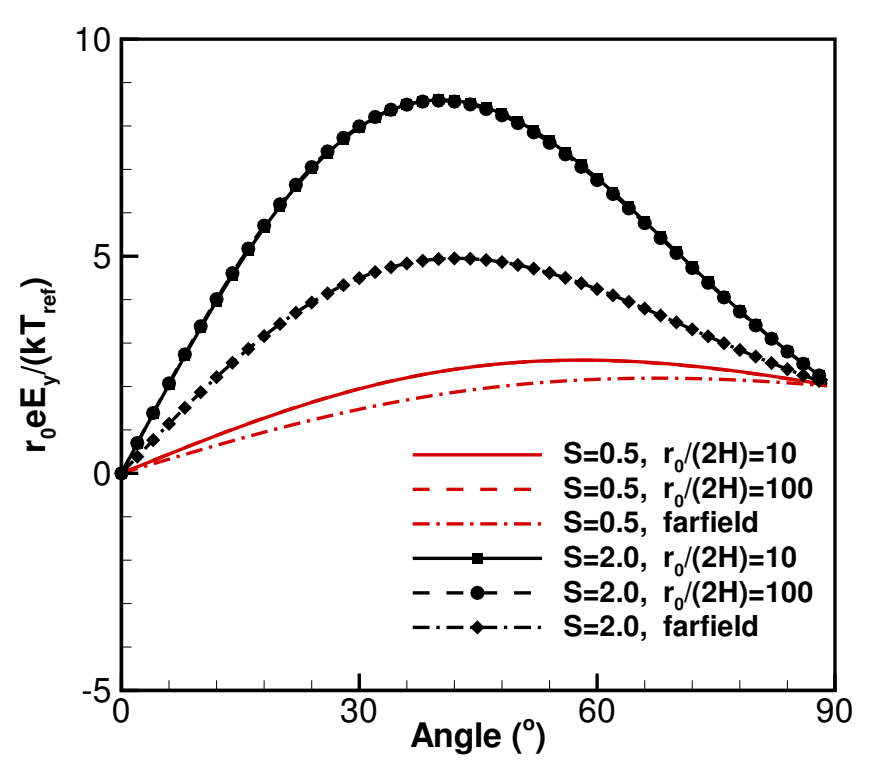

(a)

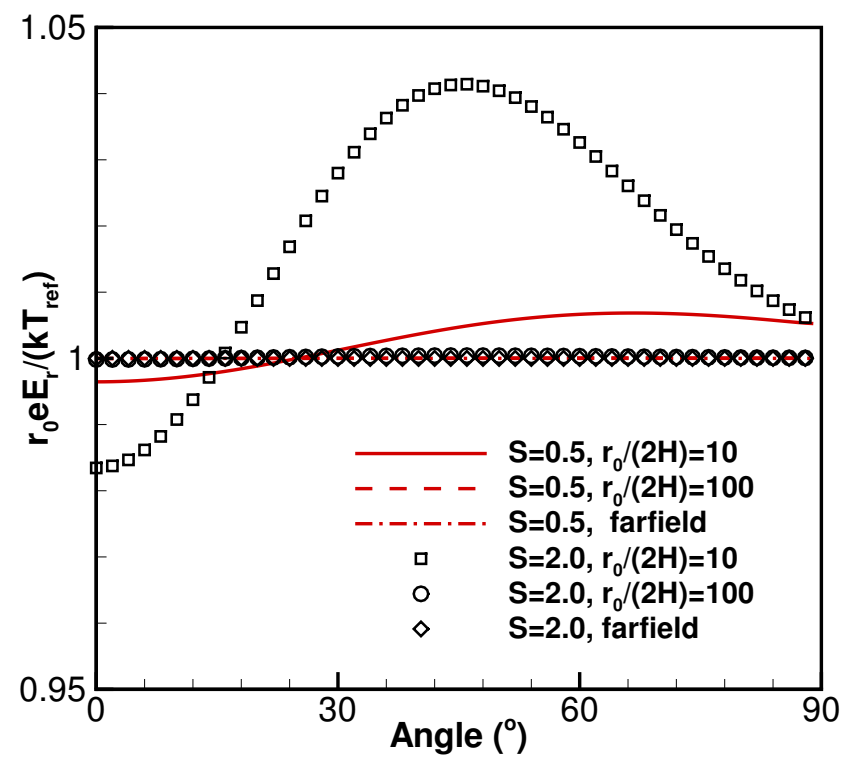

(b)

Figure 6. (a) Normalized electric field component, $e r_{0} E_{y} /\left(k T_{0}\right)$, at different angles; (b) Normalized electric field component, $e r_{0} E_{r} /\left(k T_{0}\right)$, at different angles.

Figure $6 \mathrm{~b}$ presents the normalized farfield $E_{r}\left(r, \alpha_{0}\right)$ variations. It includes six curves with different exit speed ratios $S_{0}=0.5$ and 2.0, $r_{0}=0.5$ and 2.0, Equations (14) and (22) are used for computation. As shown, the variations are very small, close to the unity as they shall be. As shown, at a location relatively closer to the exit, $r_{0} /(2 \mathrm{H})=10$, the $S_{0}=0.5$ and $S_{0}=2.0$ curves are slightly different from the unit value of 1.0. At a place relatively far away from the exit, $r_{0} /(2 \mathrm{H})=100$ and farfield, the values are unit as described Figure 6a.

Equations (16)-(22) can be used to quickly estimate the farfield properties, and the crucial factors are the specific speed ratio, $S_{0}$, and the geometry locations. A calculator may be sufficient to compute a farfield property, and there is no need of a particle simulation. However, it shall be kept in mind that those farfield simplified solutions are the approximations to the exact solutions-certain deviations may exit.

The results in this work can conveniently explain many experimental measurements as well. More accurate analysis is needed in the future to better explain the plasma plume flow results because the current work is for jet from a planar slit or a crack, while the plasma plume flows in space are axisymmetric from a round exit.

In the end, we want to explain the relations between this paper and another recently published paper [12]. Both focus on highly dilute slightly charged jet out of an exit; this paper is scenario from a planar exit, and the other is the scenario from a planar exit. The latter [12] includes more details and can aid readers understand the work. For highly dilute neutral gaseous jets out of an exit, there are another two past papers [8,9] which can help understand the work presented in this work - the first one is from a planar exit, and the other is from a round exit.

\section{Conclusions}

The work concentrated on the electric fields related with highly rarefied weakly ionized gas. The work is based on collisionless neutral gaseous jet and the Boltzmann 
relation. The analytical solutions and approximate expressions at farfield (i.e., large $r /(2 \mathrm{H})$ ) are derived and are compared.

The simplified far field solutions illustrate insights which is not evident in the corresponding exact solutions. For example, Equation (17) indicates at farfield the streamlines are straight, and the two components are constant; however, the corresponding exact solutions, Equations (2) and (3), can not offer such information.

Evidently, this new model offers a full set of exact solutions to the flow and electric fields in front of a nozzle, we shall keep in mind that they are based on neutral gas solutions, and the simplest potential relation, i.e., the Boltzmann relation is adopted to aid the study. This model is crude; however, thorough validations can be performed in a systematic manner in the future.

Author Contributions: Conceptualization, methodology, C.C.; formal analysis, validations, investigations, data curation: S.C. and C.C.; Writing-original draft preparation and visulation, S.C.; writing-review and editing and supervision, project administration, C.C. All authors have read and agreed to the published version of the manuscript.

Funding: This research received no external funding.

Conflicts of Interest: The authors declare no conflict of interest.

\section{References}

1. Pillay, S.; Singh, S.; Bharuthram, R.; Yu, M. Self-similar expansion of dusty plasmas. J. Plasma Phys. 1997, 58, 467-474. [CrossRef]

2. Lonngren, K. Expansion of a dusty plasma into a vacuum. Planet Space Sci. 1990, 38, 1457-1459. [CrossRef]

3. Birch, P.; Chapman, S. Two dimensional Particle-In-Cell simulations of the lunar wave. Phys. Plasmas 2002, 9, 1785-1789. [CrossRef]

4. Woronowicz, M. Highlights of transient plume impingement model validation and applications. In Proceedings of the 42nd AIAA Thermophysics Conference, Honolulu, HI, USA, 27-30 June 2011; AIAA Paper 2011-3772.

5. Bird, G.A. Molecular Gas Dynamics and the Direct Simulation of Gas Flows, 2nd ed.; Claredon Press: Oxford, UK, 1994.

6. Birdsall, C.; Langdon, A. Plasma Physics via Computer Simulations; Taylor \& Francis: Boca Raton, FL, USA, 2005.

7. Roy, R.I.S.; Hastings, D.; Gastonis, N.A. Ion-thruster plume modeling for back-flow contamination. J. Spacecr. Rocket. 1996, 22, 525-534.

8. Cai, C.; Zou, C. Gaskinetic solutions for high Knudsen number planar jet impingement flows. Commun. Comput. Phys. 2013, 14, 960-978. [CrossRef]

9. Cai, C.; He, X.; Zhang, K. Comprehensive studies on rarefied jet and jet impingement flows with gaskinetic methods. Commun. Comput. Phys. 2017, 23, 712-741. [CrossRef]

10. Terhune, K.J.; King, L.B.; Hause, M.L.; Prince, B.D.; Jain, N.; Hawkett, B.S. Species measurements in the beam of an ionic liquid ferro-fluid electro-spray source. In Proceedings of the 52nd AIAA/SAW/ASEE Joint Propulsion Conference, AIAA Propulsion and Energy Forum, Cleveland, OH, USA, 28-30 July 2014; AIAA Paper 2016-4550.

11. King, L.; Meyer, E.; Hopkins, M.; Hawkett, B.; Jain, N. Self-assembling array of magneto-electrostatic jets from the surface of a super-paramagnetic ionic liquid. Langmuir 2014, 30, 141443-141450. [CrossRef] [PubMed]

12. Cai, S.; Cai, C.; Li, J. Weakly charged round micro-plasma jet flows into vacuum. Phys. Plasma 2019, 26. [CrossRef]

13. Woods, F. Advanced Calculus, 2nd ed.; Ginn and Company: Oxford, UK, 1926.

14. Miller, S.; Prince, B.; Rovey, J. Capillary extraction of the ionic liquid [Bmim][DCA] for variable flow rate operations. In Proceedings of the 48th AIAA/ASME/SAE/ASEE Joint Propulsion Conference \& Exhibit, Atlanta, GA, USA, 30 July-1 August 2012; AIAA Paper 2012-3738. 